\title{
Análise comparativa entre a vascularização ventricular e do nó sinoatrial em gatos ${ }^{1}$
}

\author{
Caio Biasi ${ }^{2}$, Vicente Borelli ${ }^{3}$, Hildebrando G. Benedicto ${ }^{4}$, Miler R. Pereira ${ }^{5}$, \\ Phelipe 0. Favaron ${ }^{5}$ e Pedro P. Bombonato ${ }^{6}$
}

\begin{abstract}
Biasi C., Borelli V., Benedicto H.G., Pereira M.R., Favaron P.O. \& Bombonato P.P. 2012. [Comparative analysis between ventricular and sinoatrial node vascularization in cats.] Análise comparativa entre a vascularização ventricular e do nó sinoatrial em gatos. Pesquisa Veterinária Brasileira 31(1):78-82. Setor de Anatomia dos Animais Domésticos e Silvestres, Faculdade de Medicina Veterinária e Zootecnia, Universidade de São Paulo, Av. Prof. Dr. Orlando Marques de Paiva 87, Cidade Universitária, São Paulo, SP 05508-000, Brazil. E-mail: caiobiasi@usp.br

The possible existence of interdependence in the blood nutrition of both atrial and ventricular territories has been a subject of concern to cardiologists, mainly related to vascularization of the sinoatrial node and its dependence on just one coronary artery or both, and its relation with the predominance of these vessels in the ventricular vascularization. Therefore, this research aimed evaluated the relation of blood irrigation of the sinoatrial node in relation to the coronary artery predominance in the ventricle vascularization. In doing so, we analyzed 30 hearts of cats without pedigree, males and females, adults of several ages. They were not carrying any heart problems. The hearts were injected by the thoracic aorta with Neoprene Latex 450, stained with red pigment, and then they were dissected. It was found that when there was a prevalence of ventricular vascularization of the left type (63.34\%) the sinoatrial node irrigation was predominantly in the dependency of the Ramus proximalis atrii dextri (78.9\%) or with less frequency by Ramus proximalis atrii sinister (21.1\%). In the ventricular vascularization of the balanced type (33.34\%), the pacemaker irrigation was in dependence more often of Ramus proximalis atrii dextri $(80 \%)$ or with less frequency the nutrition of the sinoatrial node occurred by Ramus proximalis atril sinister (20\%). In a single-case, we observed the ventricular vascularization of the right type (3.34\%), the pacemaker nutrition was in an exclusive dependence of the Ramus intermedius atril dextri. These results suggest in this species there is no relationship between both the sinoatrial node irrigation and the type of ventricular vascularization, regardless of gender.
\end{abstract}

INDEX TERMS: Cats, cardiac conduction system, heart vascularization.

\footnotetext{
${ }^{1}$ Recebido em 6 de julho de 2011.

Aceito para publicação em 12 de setembro de 2011.

${ }^{2}$ Pós-Graduando em Anatomia dos Animais Domésticos e Silvestres, Departamento. de Cirurgia, Faculdade de Medicina Veterinária e Zootecnia (FMVZ), Universidade de São Paulo (USP), Av. Prof. Orlando Marques de Paiva 87, Cidade Universitária, São Paulo, SP 05508-900, Brasil. *Autor para correspondência: caiobiasi@usp.br

${ }^{3}$ Instituto de Ciências da Saúde da Universidade Paulista (Unip), Rua Doutor Bacelar 1212, Vila Clementino, São Paulo, SP 04026-002.

${ }^{4}$ Departamento de Morfologia do Instituto Biomédico, Universidade Federal Fluminense (UFF), Rua Ernani Pires de Mello 101, São Domingos, Niterói, RJ 24210-130, Brasil.

${ }^{5}$ Pós-Graduando em Anatomia dos Animais Domésticos e Silvestres, Departamento de Cirurgia, FMVZ-USP, São Paulo, SP.

${ }^{6}$ Departamento de Cirurgia, FMVZ-USP, São Paulo, SP.
}

RESUMO.- A possível existência de interdependência na nutrição de territórios atriais e ventriculares tem sido objeto de preocupação por partes dos cardiologistas, especialmente no que tange a vascularização do nó sinoatrial e sua dependência apenas de uma artéria coronária ou de ambas e de sua relação com o predomínio destes vasos na vascularização ventricular. Assim, este estudo objetiva avaliar a relação da irrigação do nó sinoatrial e a origem e a predominância das artérias coronárias na vascularização dos ventrículos, para tanto utilizou-se 30 corações de gatos sem raça definida adultos, machos e fêmeas, sem sinais de afecção cardíaca. Os corações foram injetados pela aorta torácica com Neoprene Latex 450, corados com pigmento 
vermelho e dissecados posteriormente. Verificou-se que quando ocorria predomínio da vascularização ventricular do tipo esquerda $(63,34 \%)$ a irrigação do nó sinoatrial ficou predominantemente na dependência do ramo proximal atrial direito $(78,9 \%)$ ou com menor freqüência pelo ramo proximal atrial esquerdo $(21,1 \%)$. Na vascularização ventricular do tipo equilibrada $(33,34 \%)$, a irrigação do sinoatrial ficou na dependência mais freqüentemente do ramo proximal atrial direito $(80 \%)$, ou com menor freqüência a nutrição do nó se deu pelo ramo proximal atrial esquerdo (20\%). Em um caso isolado, ocorreu a vascularização ventricular do tipo direita $(3,34 \%)$, a nutrição do sinoatrial, ficou na dependência exclusiva do ramo intermédio atrial direito. Estes resultados indicam que nesta espécie não existe relação entre a irrigação do nó sinoatrial e o tipo de vascularização ventricular, independentemente do sexo.

TERMOS DE INDEXAÇÃO: Felinos, sistema de condução, vascularização cardíaca.

\section{INTRODUÇÃ̃o}

Os tipos de vascularização ventricular, descrito por Banchi em 1904, após estudar corações humanos se encaixavam em três tipos fundamentais, isto é, o equilibrado, o direito e o esquerdo. No primeiro caso, a artéria coronária direita fornece o ramo interventricular "póstero-direito" terminal, e assim divide com a coronária esquerda a irrigação da face "póstero-direita" do coração. No tipo direito a artéria coronária direita, muito desenvolvida, depois de ceder a artéria interventricular "póstero-direita" alcança o sulco interventricular percorrendo até a margem "póstero-esquerda" do coração. Finalmente, no tipo esquerdo, a artéria coronária direita apresenta-se pouco desenvolvida e termina após ultrapassar a margem "antero-direita" do coração, cabendo à artéria coronária esquerda a irrigação de quase toda a face "póstero-direita" do órgão.

Na literatura especializada encontramos vários pesquisadores como James (1960), Blunk \& DiDio (1971), Hromada et al. (1971), Oliveira (1971) e Kronzon et al. (1974), que utilizando diferentes técnicas, tem analisado e comparado, os critérios apresentados por Banchi (1904), no referente aos tipos de vascularização arterial do coração humano, principalmente pela importância destes conhecimentos. 0 mesmo poderíamos dizer que vem acontecendo em relação aos mamíferos, estudos sobre a origem e distribuição dos ramos interventriculares são encontrados em diferentes espécies de animais (Borelli \& Fernandes Filho 1970, Borelli \& Santis Prada, 1970, Borelli et al. 1971, Andretto et al. 1973).

O nó sinoatrial, descrito por Keith e Flack em 1907, localizado entre o átrio direito e a desembocadura da veia cava cranial, é considerado o "marcapasso" do coração, porquanto coordena o seu sistema excito-condutor. Está intimamente associado com uma artéria bem definida, vale dizer a artéria nodal que é seu principal suprimento arterial, em geral, com origem unilateral (James 1961) e ponto de referência para localização deste nó em mamíferos (Keith \& Flack 1907, James 1961, Madarim-de-Lacerda 1990).
A importância funcional do nó sinoatrial tem levado inúmeros pesquisadores a se interessarem pelos aspectos morfológicos, funcionais e seu suprimento sanguíneo em diversas espécies animais como, por exemplo, em gatos (Borelli 1967), em cães (Pina et al. 1975, Randi 1988, Ovèina 2002), em bovinos (Borelli 1968, Caetano 1982, Severino 1986, Caetano et al. 1995, Ovèina 2002), em babuínos (Mandarim-de-Lacerda \& Hureau 1986), em suínos (Weaver et al. 1986, Severino et al. 2000), em ratos (Melo 2000, Melo et al. 2002) e mesmo no homem (Jatene et al. 1999, Berdajs et al. 2003). Contudo no que diz respeito à interdependência entre a irrigação do nó sinoatrial e a vascularização do miocárdio ventricular a literatura é escassa.

Baseados nestes pressupostos, este trabalho objetivou avaliar se existe relação entre a irrigação do nó sinoatrial e a vascularização dos ventrículos, em corações de gato. Procuramos enfocar em especial se o predomínio de uma das artérias coronárias na irrigação dos ventrículos corresponde ao predomínio de colaterais desta mesma coronária na irrigação do nó sinoatrial.

\section{MATERIAL E MÉTODOS}

Foram utilizados 30 corações de gatos, sem raça definida, obtidos após eutanásia que apresentavam outras afecções, excluindo os animais portadores de afecções cardíacas, sendo 11 machos e 19 fêmeas, adultos com diferentes e não conhecidas idades, procedentes do Hospital Veterinário da Universidade Paulista em São Paulo.

Após isolamento do órgão e retirada do saco pericárdico, cada coração foi lavado em água corrente e submetido à massagem manual para melhor esvaziamento das câmaras cardíacas e vasos sanguíneos. As artérias coronárias foram injetadas, de uma só vez, pela aorta torácica, canulada próxima a sua origem, com solução de Neoprene Látex 450 (Du Pont do Brasil S/A - Indústrias Químicas, São Paulo), corado em vermelho.

Após as injeções com Neoprene Látex, os corações foram fixados em solução aquosa de formol a $10 \%$ por no mínimo 72 horas para posterior dissecação da circulação ventricular e atrial com o auxílio de lupa Wild. A nomenclatura adotada para identificação e descrição das artérias atriais baseou-se no proposto por Habermehl (1959) para gatos.

Fotografias foram tiradas para documentação do trabalho e estudos estatísticos foram efetuados, com o auxílio do Teste de Qui-quadrado com nível de significância de 5\%.

\section{RESULTADOS}

A injeção de Neoprene Látex, seguida de dissecação, permitiu identificação dos tipos de vascularização ventricular e a visualização do vaso que irriga o nó sinoatrial. Quando predominou a vascularização do ventrículo através da artéria coronária esquerda (Fig.1A), em 19 casos (63,34\%), ou seja, a artéria coronária esquerda fornecia o ramo interventricular paraconal, observado a percorrer todo o sulco interventricular esquerdo, e o ramo circunflexo esquerdo que se continuava como ramo interventricular subsinuoso visto a ocupar total ou parcialmente o sulco interventricular direito, enquanto a artéria coronária direita era representada apenas pelo seu ramo circunflexo direito, que poucas vezes alcançava, apenas parcialmente, o sulco interventricular direito, a irrigação do "marca-passo" cardíaco ficava na dependência do ramo proximal atrial direito (Fig.1B) ou 

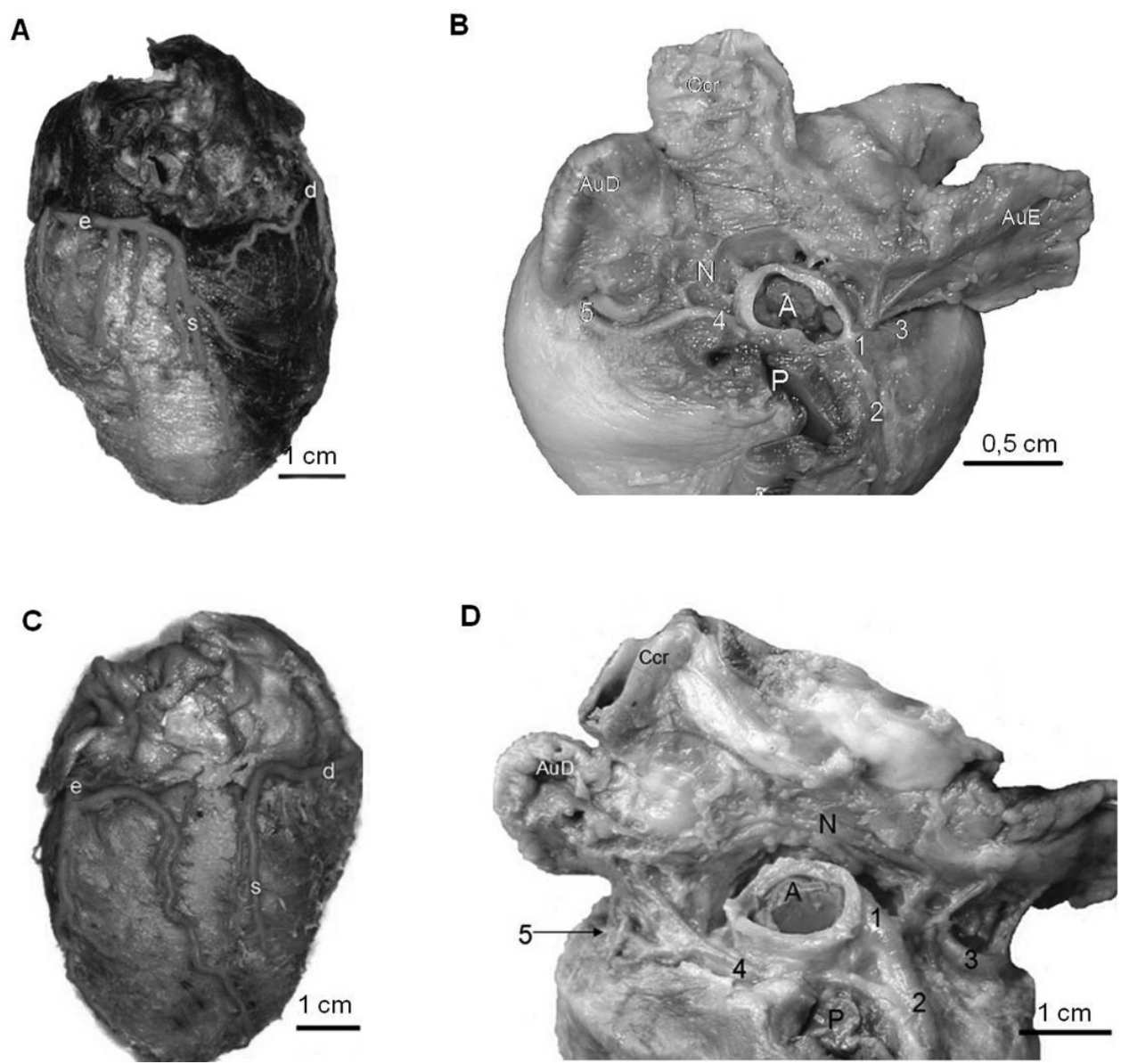

Fig.1. (A) Face atrial de coração de gato: (d) Ramo circunflexo direito, (e) ramo circunflexo esquerdo, (s) ramo interventricular subsinuoso. (B) Face auricular de coração de gato: (A) Aorta, (AuD) aurícula direita, (AuE) aurícula esquerda, (Ccr) veia cava cranial, $(\mathrm{N})$ ramo proximal atrial direito, (P) tronco pulmonar, (1) artéria coronária esquerda, (2) ramo interventricular paraconal, (3) ramo circunflexo esquerdo, (4) artéria coronária direita, (5) ramo circunflexo direito. (C) Face atrial de coração de gato: (d) Ramo circunflexo direito, (e) ramo circunflexo esquerdo, (s) ramo interventricular subsinuoso. (D) Face auricular de coração de gato: (A) Aorta, (AuD) aurícula direita, (Ccr) veia cava cranial, (N) ramo proximal atrial esquerdo, (P) Tronco pulmonar, (1) Artéria coronária esquerda, (2) ramo interventricular paraconal (3) ramo circunflexo esquerdo, (4) artéria coronária direita, (5) ramo circunflexo direito.

de colateral deste vaso, em 15 casos $(78,9 \%)$, ou do ramo proximal atrial esquerdo (Fig.1D) ou de colateral deste vaso, 4 casos $(21,1 \%)$.

Quando a nutrição dos ventrículos existia a participação equilibrada (Fig.1C), em 10 casos (33,34\%), ou seja, a artéria coronária esquerda fornecia os ramos interventricular paraconal e o circunflexo esquerdo visto a ocuparem por ordem, os sulcos interventricular esquerdo e o coronário esquerdo, enquanto que a artéria coronária direita fornecia os ramos circunflexo direito e o interventricular subsinuoso que percorriam, respectivamente os sulcos coronário direito e interventricular direito, a vascularização da região ocupada pelo nó sinoatrial ficava na dependência, mais freqüentemente do ramo proximal atrial direito ou de colateral deste vaso, em 8 casos (80\%), ou do ramo proximal atrial esquerdo ou de colateral deste vaso, em 2 casos (20\%).

Finalmente, no caso que existia predomínio da artéria coronária direita (Fig.2A) na vascularização dos ventrí- culos, 1 caso $(3,34 \%)$, ou seja, a artéria coronária direita fornecia os ramos circunflexo direito e o interventricular subsinuoso que ocupavam seus respectivos sulcos, todavia como continuação, o ramo circunflexo direito ganhava parte do sulco coronário esquerdo percorrendo-o por cerca de um terço, o território comumente reservado ao ramo circunflexo esquerdo, a irrigação do nó sinoatrial ficou na dependência exclusiva do ramo intermédio atrial direito (Fig.2B).

No respeitante à análise estatística não identificamos diferenças significativas entre os achados em relação ao sexo.

\section{DISCUSSÃO}

Inicialmente devemos destacar que apesar da importância do nó sinoatrial, a preponderância dos vasos a ele destinado não foi considerada, em nenhuma espécie, para a avaliação do desempenho de cada uma das artérias coronárias na 

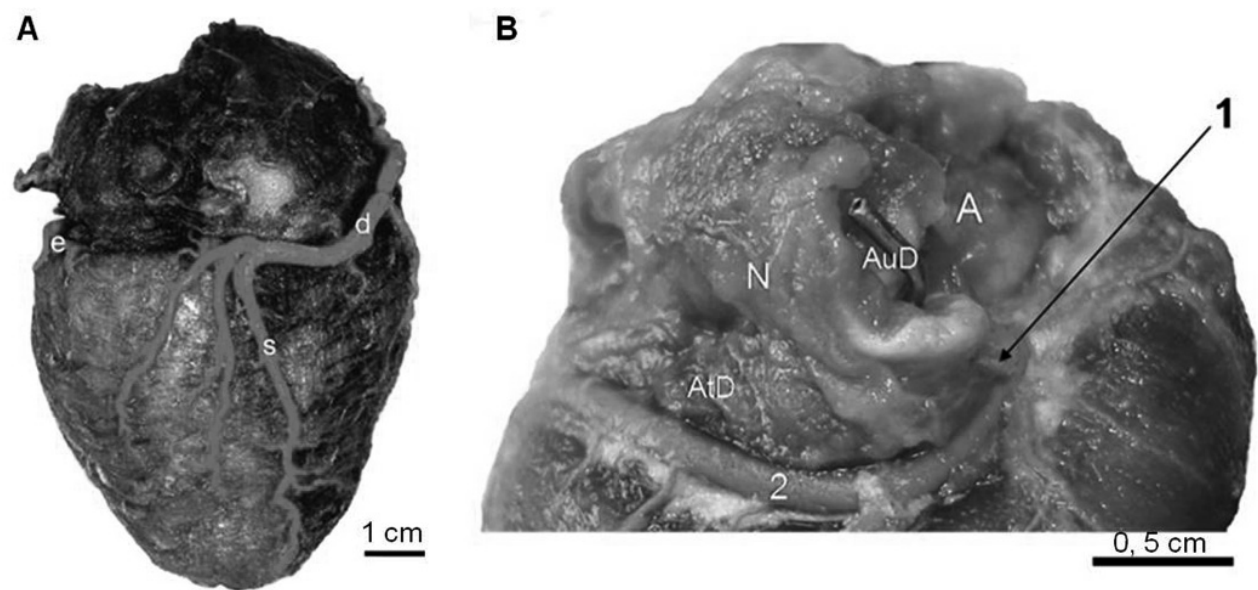

Fig.2. (A) Face atrial de coração de gato: (d) Ramo circunflexo direito, (e) ramo circunflexo esquerdo, (s) ramo interventricular subsinuoso. (B) Face cranial de coração de gato: (A) Aorta, $(\mathrm{AuD})$ aurícula direita, $(\mathrm{AtD})$ átrio direito, $(\mathrm{Ccr})$ veia cava cranial, $(\mathrm{N})$ ramo intermédio atrial direito, (1) ramo proximal atrial direito, (2) ramo circunflexo direito.

nutrição do coração, apenas verificou-se o comportamento dos vasos coronários na superfície do órgão.

De fato, especificamente ao gato, a área ocupada pelo nó sinoatrial (Keith \& Flack 1907, Chiodi 1932) fica mais comumente na dependência de colaterais do ramo circunflexo direito ou da artéria coronária direita como observado nesta espécie (Habermehl 1959, Borelli, 1967), em outras espécies domésticas (Weaver et al. 1986, Severino et al. 2000, Vidotti et al 2008) e no homem (James 1977, Nerantzis \& Avgoustakis 1980, Kyriakidis et al. 1983, Eliska \& Eliskova 1990, Didio et al. 1995, Jatene et al. 1999, Berdajs et al. 2003), ainda que com freqüências diferentes daquelas por nós identificadas, sugerindo que a artéria coronária direita, com diferentes graus de participação, responde por significativa parcela na nutrição do nó sinoatrial, independentemente da espécie animal.

Observamos que o principal vaso que nutria o nó sinoatrial, foi o ramo proximal atrial direito, concordando com o apontado nesta espécie, por Habermehl (1959) e Borelli (1967). A irrigação do nó sinoatrial a partir de ramos correspondentes do ramo proximal atrial direito, foi também observada por outros autores, ainda que tenham adotado nomenclatura diversa: para suínos (Vidotti etal. 2008) e para o homem (Krupa 1993, Sow et al. 1996, Jatene et al. 1999, Berdajs et al. 2003).

Em alguns casos, a irrigação do nó sinoatrial se fazia a partir da artéria coronária esquerda, confirmando o observado em felinos (Habermehl 1959, Borelli 1967), para cães (Pina et al. 1975, Randi 1988, Ovèina 2002), bovinos (Borelli 1968, Caetano 1982, Severino 1986, Ovèina 2002) e para o homem (James 1961, Nerantzis \& Avgoutakis 1980, Kyriakidis et al. 1983, DiDio et al. 1995, Berdajs et al. 2003).

Em nossos resultados, o vaso oriundo da artéria coronária esquerda que irrigava o nó sinoatrial, foi o ramo proximal atrial esquerdo como visto em gatos (Habermehl 1959, Borelli 1967) que adotaram nomenclatura semelhante a nossa. Este padrão foi também verificado em suínos (Vidotti et al. 2008) e em humanos (DiDio et al. 1995, Krupa 1995, Jatene et al. 1999, Futami et al. 2003, Kawashima \& Sasaki 2003) que adotaram nomenclatura diferenciada.
Quanto ao desempenho de cada artéria coronária na vascularização ventricular, padronizado por Banchi em 1904, identificamos que no gato ocorreram os três tipos de circulação, isto é, o predominantemente direito, equilibrado e esquerdo, sendo o mais freqüente o tipo esquerdo, como observado em outras espécies (Candaele \& Ghys 1958, Marques 1962).

Entretanto, apesar das várias informações encontradas na literatura, como acabamos de demonstrar, nenhum autor analisou em conjunto a vascularização do nó sinoatrial com a nutrição ventricular que foi avaliada por nós neste trabalho e observamos que o tipo mais freqüente de vascularização cardíaca foi o esquerdo porém a região ocupada pelo nó sinoatrial ficou comumente na dependência de ramos da artéria coronária direita. Quando existiu a participação equilibrada das artérias coronárias na nutrição dos ventrículos, identificamos também a preponderância da artéria coronária direita na nutrição do nó sinoatrial. Assim entendemos, com base neste trabalho, que para estabelecer os tipos de vascularização cardíaca seria necessário em cada espécie e mesmo em cada raça, considerar não só o comportamento dos ramos ventriculares, mas também a irrigação do nó sinoatrial, bem como, a nutrição das demais estruturas anatômicas responsáveis pela origem e condução do ritmo cardíaco. Isto posto, fica difícil entender, por exemplo, a existência de circulação balanceada, sem conhecer a participação de cada coronária na nutrição dos componentes do sistema de condução do ritmo cardíaco.

\section{CONCLUSÃO}

Os resultados encontrados permitem concluir que não há relação entre a vascularização do nó sinoatrial, ou seja sua origem em relação à origem e o tipo (predomínio da direita, da esquerda e equilibrada) de vascularização ventricular em gatos machos ou em fêmeas.

\section{REFERÊNCIAS}

Andretto R., Borelli V. \& Fernandes Filho A. 1973. Sobre a origem do Ramus descendens subsinuosus em cães. Revta Fac. Med. Vet. USP 10:5-9. 
Banchi A. 1904. Morfologia della arteriae coronariae cordis. Arch. Ital. Anat. Embriol. 3:87-164.

Berdajs D., Patonay L. \& Turina M.I. 2003. The clinical anatomy of the sinus node artery. Ann. Thorac. Surg. 76(3):732-736.

Blunk J.N. \& DiDio L.J.A. 1971. Types of coronary circulation in human hearts: "Types of balance" and "predominance" of the human coronary arteries. Ohio State Med. J. 67(7):596-607.

Borelli V. 1967. Contribuição ao estudo da irrigação arterial do nó sinu-atrial em Felis domestica. Dissertação de Mestrado, Faculdade de Medicina Veterinária e Zootecnia, USP, São Paulo. 27p.

Borelli V. 1968. Contribuição ao estudo da irrigação arterial do nó sinu-atrial em bovinos azebuados. Tese de Doutorado, Faculdade de Medicina Veterinária e Zootecnia, USP, São Paulo. 55p.

Borelli V. \& Fernandes Filho A. 1970. Sobre a origem do Ramus descendens subsinuosus em bovinos. Revta Fac. Med. Vet. Zootec. USP 8(2):367-374.

Borelli V. \& Santis Prada I.L. 1970. Sull' origini del Ramus descendens subsinuosus in bufali. Arch. Vet. Ital. 21(6):395-398.

Borelli V., Fernandes Filho A. \& Ferreira N. 1971. Sobre a origem a origem do Ramus descendens subsinuosus em gatos. Revta Fac. Med. Vet. Zoot. USP 8(3):569-573.

Caetano A.G. 1982. Contribuição ao estudo da irrigação arterial do Nodus sinuatrialis em bovinos da raça Gir. Dissertação de Mestrado, Escola Paulista de Medicina, USP, São Paulo, 63p.

Caetano A.G., Borelli V., Prates J.C. \& DiDio L.J.A. 1995. The arterial supply of the Nodus sinuatrialis in bovine Gir. Braz. J. Morphol. Sci. 12(2):81-88.

Candaele G. \& Ghys A. 1958. Quelques observations sur le coeur de l'okapi et sur sa circulation coronarienne. Bull. Soc. Roy. Zool. D’Anvers 7:1-4.

Chiodi V. 1932. Il nodo seno-atriale del cuore dei mammiferi. La Clin. Vet. 55:689-714.

DiDio L.J.A., Lopes A.C., Caetano A.C. \& Prates J.C. 1995. Variations of the origino of the artery of the sinoatrial node in normal human hearts. Surg. Radiol. Anat. 17(1):19-26.

Eliska O. \& Eliskova M. 1990. Arterial supply of the region of the coronary sinus in man. Cardiology 77(1):1-8.

Futami C., Tanuma K., Tanuma Y. \& Saito T. 2003. The arterial blood supply of the conducting system in normal human hearts. Surg. Radiol. Anat. 25(1):42-49.

Habermehl K.H. 1959. Die Blutgefässversorgung des Katzenherzens. Zbl. Vet. Med., Berlin, 6(1):655-680.

Hromada J., Voboril Z. \& Strnad L. 1971. Relationship of different types of branching of the coronary arteries of the human heart to origin of the principal atrial artery, sex and age. Fol. Morphol. 19(4):337-344.

James T.N. 1960. The arteries of the free ventricular walls in man. Anat. Rec. 136(3):371-384.

James T.N. 1961. Anatomy of the human sinus node. Anat. Rec. 141(1):109135.

James T.N. 1977. The sinus node. Am. J. Cardiol. 40(6):965-986.

Jatene M.B., Hervoso C.M., Terra R.M., Guimarães M.H., Monteiro R., Jatene F.B. \& Jatene A.D. 1999. Importância da anatomia da circulação coronária atrial na operação de Cox para controle da fibrilação atrial. Revta Bras. Cir. Cardiovasc. 14(1):32-38.

Kawashima T. \& Sasaki H. 2003. The morphological significance of the human sinuatrial nodal branch (artery). Heart Vessels 18(4):213-219.

Keith A. \& Flack M. 1907. The form and nature of muscular connections between the primary of the vertebrate heart. J. Anat. 41:172-189.
Kronzon I., Deutsch P. \& Glassman E. 1974. Length of the left main coronary artery: Its relation to the pattern of coronary arterial distribution. Am. J. Cardiol. 34(7):787-789.

Krupa U. 1993. The sinoatrial nodal artery in the human heart. Folia Morphol., Warsz, 52(1):29-37.

Krupa U. 1995. Vascularization of atria of the human heart in relation to the type of coronary ramification. Folia Morphol., Warsz, 54(2):111-122.

Kyriakidis M.K., Kourouklis C.B., Papaioannou J.T., Christakos S.G., Spanos G.P. \& Avgoutakis D.G. 1983. Sinus node coronary arteries studied with angiography. Am. J. Cardiol. 51(5):749-750.

Mandarim-de-Lacerda C.A. 1990. Anatomia do Coração: clínica e cirurgia. Revinter, Rio de Janeiro. 133p.

Mandarim-de-Lacerda C.A. \& Hureau U.J. 1986. Arterial vascularization of the sino-atrial and atrio-ventricular nodes and frequency of coronary artery branches in Papio erxleben, 1777. Folia Primatol., Basel, 46(2):6569.

Marques P. 1962. Alguns Aspectos Morfológicos Comparatives do Coração dos Mamíferos Domésticos. Tipografia Nunes, Lisboa. 320p.

Melo S.R. 2000. Estudo estereológico, histológico e ultra-estrutural do nó sinoatrial em ratos adultos e velhos. Tese de Doutorado, Instituto de Ciências Biomédicas, USP, São Paulo. 64p.

Melo S.R., Mandarim-de-Lacerda C.A. \& Sousa R.R. 2002. Características ultra-estruturais do nó sinoatrial de rato Wistar. Acta Sci. 24(3):681685.

Nerantzis C. \& Avgoutakis D. 1980. Na S-shaped atrial artery supplying the sinus node área: an anatomical study. Chest 78(2):274-278.

Oliveira J.A.M. 1971. Distribuição das artérias coronárias nos ventrículos do coração. Padronização da técnica para delimitação topográfica e ponderal do miocárdio dependente de cada uma. Arq. Bras. Cardiol. 24:23-29.

Ovèina F. 2002. Vascularization of the sinoatrial segment in the heart conduction system in bovine and canine hearts. Med. Arch. 56(3):123125.

Pina J.A.E., Pereira A.T. \& Ferreira A. 1975. Dos S. travaux originaux, original works: Vascularisation arterielle du noeud sino-auricular du coeur chez le chien [Arterial vascularization of the sino-auricular node of the heart in dogs]. Acta Cardiol. 30(2):67-77.

Randi R.E. 1988. Contribuição ao estudo da irrigação do nó sinoatrial em cães da raça Boxer. Tese de Doutorado, Faculdade de Medicina Veterinária e Zootecnia, USP, São Paulo. 66p.

Severino R.S. 1986. Contribuição ao estudo da vascularização arterial do nó sinoatrial em bovinos da raça Indubrasil. Dissertação de Mestrado, Faculdade de Medicina Veterinária e Zootecnia, USP, São Paulo. 66p.

Severino R.S., Carneiro e Silva F.O., Drummond S.S., Bombonato P.P. \& Alves F.P. 2000. Irrigação do nó sinoatrial em suínos da raça Piètrain. Braz. J. Vet. Res. Anim. Sci. 37(4):13-16.

Sow M.L., Ndoye J.M. \& Lo E.A. 1996. The artery of the sinoatrial node: anatomic consideration based on 45 injection-dissection of the heart. Surg. Radiol. Anat. 18(2):103-109.

Vidotti A.P., Agreste F.R., Bombonato P.B., Prado I. M.M. \& Monteiro R. 2008. Vascularização arterial da região do nó sinoatrial em corações de suínos: origem, distribuição e quantificação. Pesq. Vet. Bras. 28(2):113118.

Weaver M.E., Pantely G.A., Bristow J.D. \& Ladley H.D. 1986. A quantitative study of the anatomy and distribution of coronary arteries in swine in comparison with other animals and man. Cardiovasc. Res. 20(12):907917. 